\title{
Circular Polarization Hologram Realized by Pancharatnam-Berry Phase in Microwave Frequency
}

\author{
Tao Yan, Qian Ma, Tiejun Cui \\ State Key Laboratory of Millimeter Waves, Southeast University, Nanjing, China \\ Email: tjcui@seu.edu.cn
}

How to cite this paper: Yan, T., Ma, Q. and Cui, T.J. (2020) Circular Polarization Hologram Realized by Pancharatnam-Berry Phase in Microwave Frequency. Journal of Computer and Communications, 8, 134-141. https://doi.org/10.4236/jcc.2020.812013

Received: November 30, 2020 Accepted: December 17, 2020 Published: December 24, 2020

\begin{abstract}
Metasurfaces have shown great potential in controlling the propagation of electromagnetic waves, making them suitable for holographic beam-shaping elements. Using the principle of array synthesis, holograms have been realized in microwave frequency to manipulate electromagnetic waves to generate specific field patterns. However, current research is almost based on linear polarization and has little analysis on the circular polarization microwave hologram. Herein, we propose a circular polarization hologram realized by sub-wavelength units with self-adaptively phase profiles of electromagnetic waves. The full, continuous control of the phase is achieved by using a single structure unit based on the Pancharatnam-Berry phase. The inhomogeneity of wave-front is considered through amplitude and phase compensation which are essential to improve the quality of microwave hologram. The model achieves circular polarization hologram in microwave frequency and the clear field pattern is generated. The circular polarization hologram enriched the research field of microwave imaging, thus providing more inspirations for the development of various related techniques.
\end{abstract}

\section{Keywords}

Metasurfaces, Circular Polarization Hologram, Pancharatnam-Berry Phase, Microwave Hologram

\section{Introduction}

The hologram is a technique that reconstructs the wave-front of electromagnetic (EM) waves to display object pattern on the monitor area. The holography principle has become a universal and simple approach to solve the inverse engineer- 
ing problems for electromagnetic waves [1]. Microwave hologram is a feasible way to manipulate EM waves which have raised many interests [2]-[8]. However, conventional microwave holographic techniques are always established in the situation of linear polarization, and rarely involve circular polarization holograms which also play an important role in many functions.

Metasurfaces, within ultrathin and sub-wavelength features, are applied to realize plenty of functions such as abnormal refraction, beam forming, polarization conversion, and hologram [9]-[15]. Phase-only metasurfaces, which are powerful tools to control the beam properties, have the ability to perform different functions [2] [7]. The metasurfaces used in microwave hologram always utilize the phase responses of linear polarization EM waves. Through a transmission metasurface, a linear polarization multiplexing hologram has been designed, and clear field patterns are presented [6]. Huygens metasurface has been designed to the modulation of focal energy distribution with high energy efficiency by using holography theory [3]. Electromagnetic reprogrammable coding-metasurface holograms have also been designed to change the dynamic object field patterns [2] [7]. Circular polarization hologram has been utilized to fulfill multi-focus and point imaging [5]. Most microwave holograms are designed with linear polarization while the circular polarization always seems to have a bad effect.

In this article, we design a circular polarization hologram based on the Pancharatnam-Berry (P-B) phase metasurface to realize the letter hologram [16] [17] [18]. The horn signal obliquely irradiates on the metasurface, and the target pattern is formed on the observation plane as shown in Figure 1. The P-B phase metasurface is regarded as a radome to manipulating the arrived EM waves. The metasurface units obtain different P-B phases by rotating the whole structure to control the circular polarization wave-front. By designing the phases in $\mathrm{x}$ - and y-polarizations differing by $\pi$, the P-B phase and rotation angle present a linear relationship which greatly simplifies the design process [19] [20]. A modified G-S algorithm, considering the inhomogeneous horn wave-front, is applied to calculate the holographic phase mask [3] [5]. The calculation methods of the

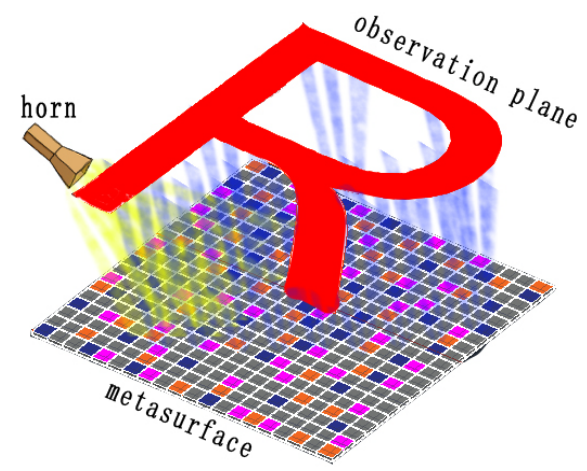

Figure 1. Schematic diagram of the reflection hologram system. The horn antenna obliquely illuminates to the metasurface, and the field pattern is formed on a plane with a certain height. 
amplitude and the phase distributions of circular polarization wave-front have also been introduced in the article.

\section{Holography Methodology}

To construct the holography system, the metasurface and the synthesis algorithm are essential to be considered. The building blocks constructing the metasurface are a set of units representing phase adjustment pixels. According to the concept of the P-B phase, we design a simple reflect unit operating at $15 \mathrm{GHz}$ as shown in Figure 2. The unit consists of two layers of metal and one layer of substrate. The substrate is a layer of FR- $4\left(\varepsilon_{r}=4.4+0.02 \mathrm{i}, h=1 \mathrm{~mm}\right)$, the unit size is $6 \mathrm{~mm} \times 6 \mathrm{~mm}(p=6 \mathrm{~mm})$, and the thickness of the copper $t=0.018 \mathrm{~mm}$. To make the unit P-B phase exhibits a linear relationship with the rotation angle, the phase difference between the $\mathrm{x}$ - and $\mathrm{y}$-polarizations is $\pi$ apart [19]. Optimized by the software of CST Microwave Studio, the metal-atom size is selected as $w=3.45 \mathrm{~mm}$ and $d=4.8 \mathrm{~mm}$. The simulation results of P-B phase change with the unit rotating angle $\theta$ are depicted in Figure 3(a) and Figure 3(b). It is clear to be observed that as the unit rotates by $\theta$, an additional $2 \theta \mathrm{P}-\mathrm{B}$ phase is added to the reflective wave with a polarization conversion. If an RCP wave irradiates to the unit, the reflection angle changes to LCP with a $2 \theta$ additional phase. In the same way, if the incident single is an LCP wave, the reflection changes to $\mathrm{RCP}$ with a $-2 \theta$ additional phase. By changing the rotation angle, the $\mathrm{P}-\mathrm{B}$ phases in the full phase range are obtained with the reflection amplitudes remain at a high level.

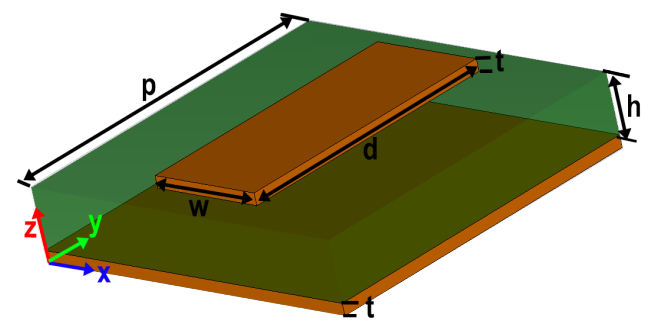

Figure 2. The structure of the unit. The unit is optimized to a $\pi$ phase difference in $\mathrm{x}$ - and y-polarizations. The specific parameters are $p=6 \mathrm{~mm}, h=1 \mathrm{~mm}, t=0.018 \mathrm{~mm}, w=3.45$ $\mathrm{mm}$, and $d=4.8 \mathrm{~mm}$.

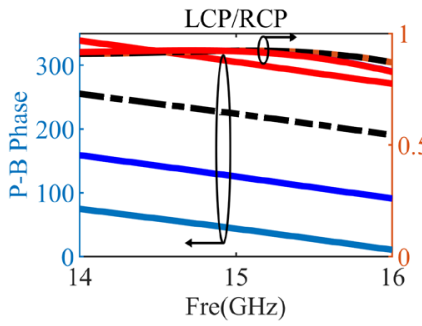

(a)

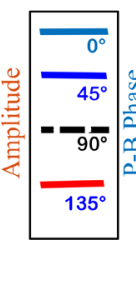

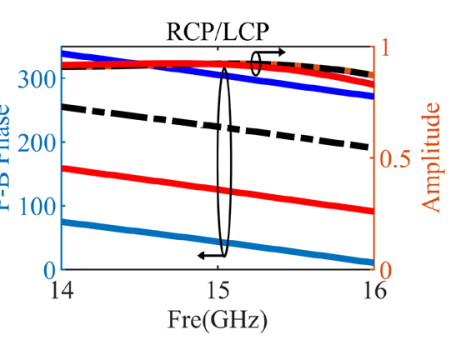

(b)

Figure 3. The P-B phasespresent a linear relationship with the rotate angle. The RCP incident wave presents a $2 \theta$ additional $\mathrm{P}-\mathrm{B}$ phase with polarization conversion, and the LCP incident wave presents a $-2 \theta$ additional $\mathrm{P}-\mathrm{B}$ phase with polarization conversion. 
A modified G-S algorithm that considers the inhomogeneous wave-front is used to calculate the holography phase mask [5] [21]. By integrating the specific radiation pattern of the feeding antenna, the algorithm considers the influence of the inhomogeneous wave-front. Because the amplitude and phase of circular polarization wave-front are hard to be obtained directly, we use a simple conversion to solve the problem. We always describe a single-direction propagation RCP wave by using $E=\left(j e_{x}+e_{y}\right) \mathrm{e}^{-j \beta z}$ while LCP described it as $E=\left(e_{x}+j e_{y}\right) e^{-j \beta z}$. So as an RCP horn antenna wave-front, the E-field intensity is $|E|=\sqrt{e_{x}^{2}+e_{y}^{2}}$, and the wave-front phase distribution is regarded as the additional phase in $\mathrm{x}$-polarization, while the LCP antenna wave-front phase distribution is the additional phase in y-polarization.

In this work, we utilize an RCP horn to design the circular polarization hologram and the wave-front singles are obtained by full-wave simulation. The metasurface lies in the xoy plane centered in the origin, the horn antenna is at the $(0,-90 \mathrm{~mm}, 90 \mathrm{~mm})$ and points to the center of the metasurface at a $45^{\circ}$ angle. The signal amplitude is calculated by $|E|=\sqrt{e_{x}^{2}+e_{y}^{2}}$ and the phase takes from $\mathrm{x}$-polarization of the circular polarization horn as depicted in Figure 4(a) and Figure 4(b).

We compensate the inhomogeneous wave-front by utilizing the amplitude and phase into the modified G-S algorithm. The algorithm takes the amplitude as the initial condition in the calculation process, and the result is compensated with the phase distribution of the wave-front to generate the final phase mask object to the metasurface as shown in Figure 5. The phase mask is $40 \times 40$ corresponding to 1600-pixel units and each unit represents an object P-B phase generated by rotating the unit with a special angle.

Combining the phase mask with the P-B phase unit, the metasurface is modeled with the object phase distribution as depicted in Figure 6. The metasurface consists of $1600(40 \times 40)$ units, and each unit is rotated by an angle to provide a P-B phase corresponding to the object phase mask.

The simulation result of circular polarization hologram obtained by CST Microwave Studio is depicted in Figure 7. The metasurface manipulates the arrived

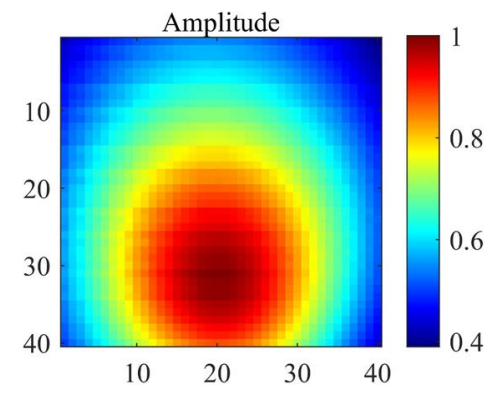

(a)

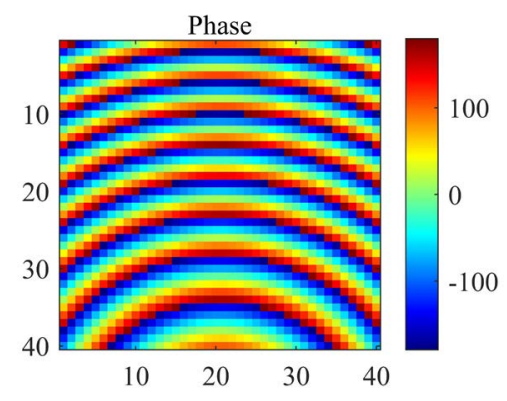

(b)

Figure 4. Amplitude and phase of the RCP polarization horn wave-front. (a) The amplitude is calculated by $|E|=\sqrt{e_{x}^{2}+e_{y}^{2}}$; (b) The phase is selected as the phase of x-polarization. 


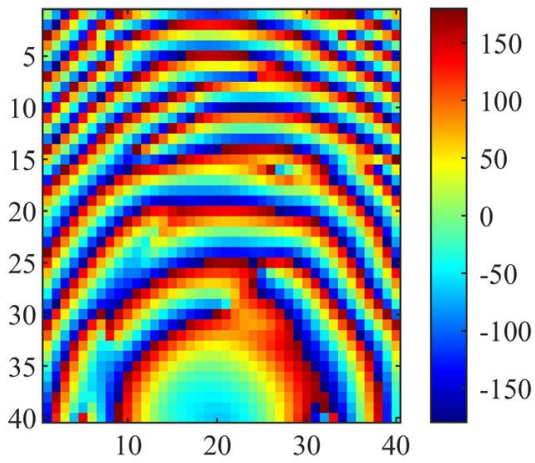

Figure 5. Phase mask calculated by the holographic algorithm which represents the object phase distribution of the metasurface.

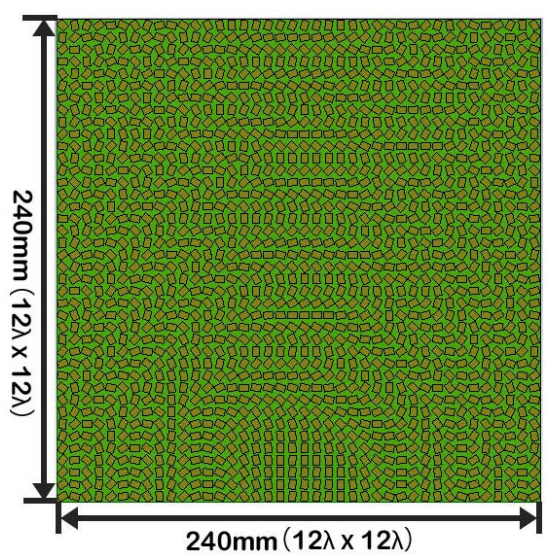

Figure 6. The metasurface model consists of $1600(40 \times 40)$ units within an area of 240 $\mathrm{mm} \times 240 \mathrm{~mm}(12 \lambda \times 12 \lambda)$.

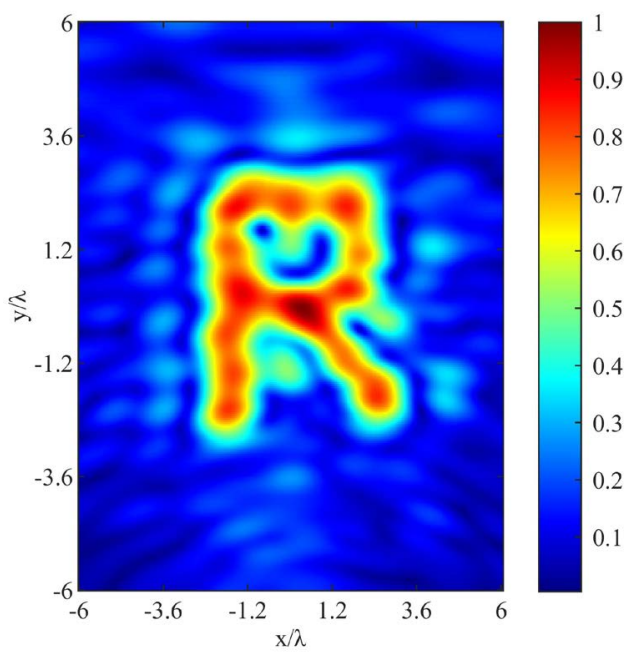

Figure 7. The simulation result of circular polarization hologram. The result obtains a clear E-field intensity pattern " $\mathrm{R}$ " in the observation plane.

EM waves to form a target field pattern in a specific area. The simple example depicts the process of obtaining the E-field intensity patterns " $R$ " using the RCP wave. With the same methodology, it can be indicated that different patterns can 
also be obtained by change the phase mask of the metasurface. The phase-only metasurface well controls the incident RCP wave and forms a specific imaging pattern on the target plane. However, the result also shows some unevenness and background energy diffusion, which is an undesirable phenomenon. The reasons may lie in the error of the P-B phase and the limitations of the GS algorithm [21] [22], but it has made progress compared with the other research in the field of circular polarization microwave hologram.

\section{Conclusions}

In this work, we succeed in utilizing the P-B phase to achieve circular polarization holograms to obtain the letter field pattern. With a simple structure of the P-B phase element, the EM wave is manipulated to utilized the scheduled function. Comparing with the previous research, we make a breakthrough in circular polarization holograms. The horn wave-front is accurately described by using the full-wave simulation software and then compensated in the design process to reduce the influence of the inhomogeneity.

The holography methodology can also be applied to any situation of the incident wave to obtain the expected patterns. This work can also be utilized to control energy distribution which may shed new light on wireless power transmission. The ability to control the far-field pattern may also be obtained assuming the hologram worked in far-field applications.

\section{Acknowledgements}

To a certain extent, this work is supported by the National Key Research and Development Program of China under Grant Nos. 2017YFA0700201, 2017YFA0700202, 017YFA07002-03, the National Science Foundation of China under Grant Nos. 61871127, 61890544, 61801117, 61735010, 61731010, 1722106, 61701107, 61701108, and 61631007, and 111 Project under Grant No.111-2-05, and Fund for International Cooperation and Exchange of the National Natural Science Foundation of China 61761136007.

\section{Conflicts of Interest}

The authors declare no conflicts of interest regarding the publication of this paper.

\section{References}

[1] Huang, L., Zhang, S. and Zentgraf, T. (2018) Metasurface Holography: From Fundamentals to Applications. Nanophotonics, 7, 1169-1190. https://doi.org/10.1515/nanoph-2017-0118

[2] Li, L., et al. (2017) Electromagnetic Reprogrammable Coding-Metasurface Holograms. Nature Communications, 8, 1-7. https://doi.org/10.1038/s41467-017-00164-9

[3] Wang, Z., et al. (2018) Huygens Metasurface Holograms with the Modulation of Focal Energy Distribution. Advanced Optical Materials, 6, Article ID: 1800121. 
https://doi.org/10.1002/adom.201800121

[4] Li, L., et al. (2019) Machine-Learning Reprogrammable Metasurface Imager. Nature Communications, 10, 1-8. https://doi.org/10.1038/s41467-019-09103-2

[5] Wang, Y., et al. (2019) Multi-Focus Hologram Utilizing Pancharatnam-Berry Phase Elements Based Metamirror. Optics Letters, 44, 2189-2192. https://doi.org/10.1364/OL.44.002189

[6] Wu, J.W., et al. (2018) Synthesis Algorithm for Near-Field Power Pattern Control and Its Experimental Verification via Metasurfaces. IEEE Transactions on Antennas and Propagation, 67, 1073-1083. https://doi.org/10.1109/TAP.2018.2882645

[7] Wan, X., et al. (2019) Multichannel Direct Transmissions of Near-Field Information. Light. Science \& Applications, 8, 1-8. https://doi.org/10.1038/s41377-019-0169-3

[8] Wu, J.W., et al. (2020) Anisotropic Metasurface Holography in 3D Space with High Resolution and Efficiency. IEEE Transactions on Antennas and Propagation. https://doi.org/10.1109/TAP.2020.3008659

[9] Ma, Q., et al. (2019) Controllable and Programmable Nonreciprocity Based on Detachable Digital Coding Metasurface. Advanced Optical Materials, 7, Article ID: 1901285. https://doi.org/10.1002/adom.201901285

[10] Chen, T., et al. (2019) Direct Current Remote Cloak for Arbitrary Objects. Light. Science \& Applications, 8, 1-6. https://doi.org/10.1038/s41377-019-0141-2

[11] Ma, Q. and Cui, T.J. (2020) Information Metamaterials: Bridging the Physical World and Digital World. PhotoniX, 1, 1-32.

https://doi.org/10.1186/s43074-020-00006-w

[12] Cui, T.J., Wu, R.Y., Wu, W., Shi, C.B. and Li, Y.B. (2017) Large-Scale Transmission-Type Multifunctional Anisotropic Coding Metasurfaces in Millimeter-Wave Frequencies. Journal of Physics D: Applied Physics, 50, Article ID: 404002. https://doi.org/10.1088/1361-6463/aa85bd

[13] Cui, T.J. (2017) Microwave Metamaterials. National Science Review, 5, 134-136. https://doi.org/10.1093/nsr/nwx133

[14] Ma, Q., et al. (2019) Smart Metasurface with Self-Adaptively Reprogrammable Functions. Light. Science Applications, 8, 1-12. https://doi.org/10.1038/s41377-019-0205-3

[15] Zhang, L., et al. (2018) Space-Time-Coding Digital Metasurfaces. Nature Communications, 9, 1-11. https://doi.org/10.1038/s41467-018-06802-0

[16] Xu, H., Liu, H., Ling, X., Sun, Y. and Yuan, F. (2017) Broadband Vortex Beam Generation Using Multimode Pancharatnam-Berry Metasurface. IEEE Transactions on Antennas and Propagation, 65, 7378-7382. https://doi.org/10.1109/TAP.2017.2761548

[17] Huang, L., et al. (2012) Dispersionless Phase Discontinuities for Controlling Light Propagation. Nano Letters, 12, 5750-5755. https://doi.org/10.1021/nl303031j

[18] Ding, X., et al. (2015) Ultrathin Pancharatnam-Berry Metasurface with Maximal Cross-Polarization Efficiency. Advanced Materials, 27, 1195-1200. https://doi.org/10.1002/adma.201405047

[19] Hasman, E., Kleiner, V., Biener, G. and Niv, A. (2003) Polarization Dependent Focusing Lens by Use of Quantized Pancharatnam-Berry Phase Diffractive Optics. Applied Physics Letters, 82, 328-330. https://doi.org/10.1063/1.1539300

[20] Bomzon, Z., Biener, G., Kleiner, V. and Hasman, E. (2002) Space-Variant Pancharatnam-Berry Phase Optical Elements with Computer-Generated Subwavelength 
Gratings. Optics Letters, 27, 1141-1143. https://doi.org/10.1364/OL.27.001141

[21] Gerchberg, R.W. (1972) A Practical Algorithm for the Determination of Phase from Image and Diffraction Plane Pictures. Optik, 35, 237-246.

[22] Fienup, J.R. (1982) Phase Retrieval Algorithms: A Comparison. Applied Optics, 21, 2758-2769. https://doi.org/10.1364/AO.21.002758 